\title{
A PRESENÇA DE MULHERES CRIA VALOR ÀS EMPRESAS?
}

\section{DOES THE PRESENCE OF WOMEN CREATE VALUE FOR COMPANIES?}

\author{
Anie Levinbuk Schmiliver \\ Bacharel em Administração pela Universidade Presbiteriana Mackenzie \\ São Paulo, SP, Brasil \\ Email: anielevisch@gmail.com \\ Marcella Scaraficci Teixeira \\ Bacharel em Administração pela Universidade Presbiteriana Mackenzie \\ São Paulo, SP, Brasil \\ Email: marcella.scaraficci@gmail.com \\ Mariana Domingues Brandão \\ Bacharel em Administração pela Universidade Presbiteriana Mackenzie \\ Acadêmica de Direito na Universidade Anhembi Morumbi \\ São Paulo, SP, Brasil \\ Email: maridbrandao@hotmail.com \\ Vitória Dantas de Andrade \\ Bacharel em Administração pela Universidade Presbiteriana Mackenzie \\ São Paulo, SP, Brasil \\ Email: vda_andrade@hotmail.com \\ Michele Nascimento Jucá \\ Professora do Centro de Ciências Sociais Aplicadas (CCSA) da Universidade Presbiteriana Mackenzie \\ São Paulo, SP, Brasil \\ Email: michele.juca@uol.com.br
}

\section{RESUMO}

Mecanismos de governança corporativa são instrumentos relevantes na criação de valor e melhoria da performance financeira das empresas, destacando-se o papel do conselho de administração e diretoria. Segundo a teoria institucional, a simples presença de mulheres no conselho administrativo e diretoria sinaliza o reconhecimento e sucesso alcançados por elas na empresa. Assim, este estudo tem por objetivo verificar as seguintes hipóteses. $\mathrm{H}_{1}$ : A presença de mulheres no conselho de administração e diretoria aumenta o valor da empresa e $\mathrm{H}_{2}$ : A presença de mulheres no conselho de administração e diretoria melhora a performance financeira da empresa. A amostra final é composta por 113 companhias brasileiras não financeiras de capital aberto, entre os anos de 2010 e 2017, listadas na Brasil, Bolsa, Balcão. A confirmação de ambas as hipóteses ocorre por meio de estatística descritiva, análise de correlação e modelo de regressão pooled cross section.

Palavras-chave: Diversidade de gênero.

Governança corporativa. Valor das empresas.

Performance financeira. Regressão cross section com dados empilhados.

\section{ABSTRACT}

Corporate governance mechanisms are important instruments in creating value and improving the financial performance of companies, especially the role of the board of directors and board of executive officers. According to institutional theory, the mere presence of women on the board and board of directors signals the recognition and success achieved by them in the company. Thus, this study aims to verify the following hypotheses. $H_{1}$ : The presence of women on the board and board increases the company's value and $\mathrm{H}_{2}$ : The presence of women on the board and board improves the company's financial performance. The final sample is composed of 113 Brazilian non-financial companies, publicly traded between 2010 and 2017, listed in Brazil, Bolsa, Balcão. The confirmation of both hypotheses occurs through descriptive statistics, correlation analysis and pooled cross section regression model.

Keywords: Gender diversity. Corporate governance. Value of companies. Financial performance. Pooled cross section regression. 


\section{INTRODUÇÃO}

O Instituto Brasileiro de Governança Corporativa (IBGC) (IBGC, 2010) define o conselho de administração como o principal órgão de governança, o qual estabelece as diretrizes da organização. De acordo com a Lei no. 6.404/76, companhias abertas e de capital autorizado devem ter, obrigatoriamente, um conselho de administração. Portanto, monitorar sua performance é um importante mecanismo de controle de governança corporativa para as empresas. Outro elemento fundamental de governança corporativa é a diretoria, responsável por coordenar os negócios da organização, implementar a estratégia definida pelo conselho de administração e respeitar as diretrizes estabelecidas por ele (IBGC, 2007). Em termos mais formais, a governança corporativa pode ser compreendida como "sistema pelo qual as organizações são dirigidas, monitoradas e incentivadas, envolvendo os relacionamentos entre proprietários, conselho de administração, diretoria e órgãos de controle" (IBGC, 2009, p. 19).

A eficácia do conselho depende de seus recursos de capital humano, tais como gênero, educação, experiências gerenciais entre outras, como explica a teoria da dependência de recursos (PFEFFER; SALANCIK, 1978). Neste contexto, surge o questionamento a respeito da diversidade de gênero nos órgãos de governança corporativa. Em outras palavras, busca-se compreender qual é a relação entre a presença de mulheres nos conselhos administrativos e diretoria e a criação de valor nas empresas. Segundo Campbell e Mínguez-Vera (2008) e Cardoso e Hanashiro (2018), a composição de gênero do conselho pode melhorar o desempenho da organização devido a um conjunto único de recursos que mulheres trazem ao negócio e, portanto, pode afetar a performance financeira da empresa. Para Adams e Ferreira (2009), diretoras do gênero feminino são mais propensas a participarem de diversos conselhos e comitês de monitoramento, em um ritmo mais acelerado, que diretores de gênero masculino. Esses são importantes mecanismos de governança corporativa que auxiliam no desempenho da organização. Por sua vez, Beauvoir (1980) sugere que a mulher em cargo de liderança se mostra escrupulosa, minuciosa e facilmente agressiva. Para a autora, essas são características que agregam valor à empresa em cargos os quais exigem habilidades de comando.

Estudos internacionais apontam para resultados divergentes sobre o tema, apresentando relações positivas, negativas e neutras entre a presença de mulheres no conselho de administração/diretoria e a criação de valor nas empresas analisadas (ADAMS; FERREIRA, 2009; NIELSEN; HUSE, 2010; KLÜCK, 2012; SUDECK; LATRIDIS, 2014; BOSCIA; MARCHETTI; STEFANELLI, 2015; VINTILÃ; ONOFREI; GHERGHINA, 2015; BYRON; POST, 2016; KIM; STARKS, 2016; COOK; GLASS, 2017). O estudo de Klück (2012), por exemplo, busca verificar se o anúncio de nomeações de mulheres para os conselhos de administração das organizações possui impacto no valor de mercado dessas empresas. Ele identifica uma relação negativa e significante no retorno das ações no período em que uma lei de cotas de gênero é anunciada na Espanha e na França, causando perda no valor dessas organizações.

Já na pesquisa em empresas alemãs de Sudeck e latridis (2014), constata-se uma relação positiva entre o valor de mercado e a presença de mulheres nos conselhos das empresas analisadas. $O$ estudo de Low, Roberts e Whiting (2015) afirma que forçar nomeações para a diretoria ou estabelecer cotas de gêneros, em países com fortes resistências culturais, pode reduzir o desempenho da organização. Nesse sentido, é possível observar que o impacto, positivo ou negativo, de mulheres nos conselhos de administração das empresas pode variar em função da cultura do país e/ou empresa, do período apurado para análise, do 
mercado, além de outros fatores, apontando para a não existência de uma verdade universal sobre o impacto da presença do sexo feminino nos conselhos e diretorias.

Por sua vez, a teoria de capital humano afirma que os conhecimentos e habilidades individuais, se bem investidos, podem beneficiar a organização (LUCENA, 2003). Essa teoria também prediz que a performance do conselho pode ser afetada pela diversidade dele, como resultado do diverso e único capital humano que cada indivíduo tem. Resultados do estudo de Kim e Starks (2016) sobre a diversidade de gênero nos conselhos mostram que a presença feminina no conselho não somente contribui com tipos mais diversos de experiência, mas também com conhecimentos singulares de especialização que não estão presentes nos conselhos corporativos vigentes. De fato, países com leis que exigem percentuais obrigatórios mínimos de participação feminina nos conselhos melhoram o desempenho de suas empresas (FERREIRA, 2010; ISIDRO; SOBRAL, 2015)

Não obstante esse fato, verifica-se que há baixa participação feminina nos conselhos de administração das grandes empresas, tanto no meio internacional (FERREIRA, 2010; DEZSO; ROSS, 2012) quanto no Brasil (SANTOS; RIBEIRO, 2009; MADALOZZO, 2011). De acordo com pesquisa realizada entre 2010 e 2012 pelo IBGC (2015), apenas 7,2\% dos membros dos conselhos administrativos são do sexo feminino. Isso ocorre ainda que haja um aumento da participação das mulheres no mercado de trabalho (DIEESE, 2013). Diante do exposto, esta pesquisa pretende responder ao seguinte problema: A presença feminina no conselho administrativo e diretoria possui impacto positivo no valor e performance financeira das empresas?

Assim, este estudo tem como objetivo verificar se a presença feminina no conselho administrativo e diretoria possui impacto positivo no valor e performance financeira das empresas. Para tanto são definidas as seguintes hipóteses: $\mathrm{H}_{1}$ : A presença de mulheres no conselho de administração e diretoria aumenta o valor da empresa e $\mathrm{H}_{2}$ : A presença de mulheres no conselho de administração e diretoria melhora a performance financeira da empresa. A amostra final é composta por 113 companhias brasileiras não financeiras de capital aberto, entre os anos de 2010 e 2017, listadas na Brasil, Bolsa, Balcão (B3). A verificação das hipóteses mencionadas ocorre por meio de estatística descritiva, análise de correlação e modelo de regressão cross section pooled.

\section{REVISÃO DA LITERATURA}

A teoria institucional discute a forma como as ações de uma organização podem ser influenciadas por fatores externos e sociais. A institucionalização pode ser entendida como decisões e atitudes diversas que, quando realizadas constantemente, trazem resultados significativos e tornam-se valores reais para as pessoas que as praticam e as reproduzem. Levando em conta a magnitude da influência que uma empresa tem sobre outra, as organizações estão sempre em busca de se adaptar ao meio em que a sociedade e as leis estão inseridas (MEYER; ROWAN, 1977; DIMAGGIO; POWELL, 1983).

Neste contexto, essa teoria possibilita analisar as mudanças institucionais que fundamentam a sociedade e, com isso, entender a participação da mulher nos conselhos e diretoria, assim como sua possível contribuição para a organização, no momento em que ela estaria ocupando um lugar tradicionalmente instituído como masculino. De fato, quando uma empresa aloca mulheres em posições de decisões 
estratégicas, ela mostra que está comprometida em ter uma diversidade de gênero em todos os níveis da sua organização (BLUM; FIELDS, 1994; DAILY; DALTON, 2003).

Blum e Fields (1994) verificam que empresas enfrentam pressões institucionais para encorajar diversidade de gênero em seus postos de gestão. A nomeação de mulheres para participação em conselhos de administração e diretoria é um meio de obter-se legitimidade na indústria, sinalizando um amplo compromisso da corporação com a diversidade. Por sua vez, Bilimoria (2000) argumenta que a simples presença de mulheres no conselho administrativo e diretoria sinaliza o reconhecimento e sucesso alcançados por elas na empresa. Isso contribui com a reputação da organização em ter uma cultura de liderança que estimula equilíbrio entre vida e carreira, facilitando o desempenho e progressão das mulheres. Já para Burke (1994), essa presença impressiona os acionistas e aqueles outros stakeholders que estão preocupados com a questão da diversidade nas empresas. Deszo e Ross (2012) complementam esse argumento ao citar que toda ação que é tomada para melhorar a performance e escolha dos representantes dessas entidades de governança deve, consequentemente, melhorar o desempenho da empresa.

Além disso, verifica-se que nas empresas há uma separação entre propriedade e gestão. Cabe aos administradores buscar a maximização da riqueza da empresa e, por conseguinte, de seus acionistas. No entanto, a teoria de agência afirma que não é possível assegurar que o agente tome sempre decisões financeiras ótimas, sob a ótica do proprietário, gerando conflitos. Para buscar conciliar os interesses de ambos, as empresas instituem mecanismos de governança corporativa, entre outras ações, gerando custos de agência (JENSEN; MECKLING; 1976)

Entre os mecanismos de governança corporativa, destaca-se a presença de um conselho de administração que tem por objetivo, entre outros, fiscalizar a gestão dos diretores, que são responsáveis pela representação da companhia e pela implementação das deliberações da assembleia geral e do próprio conselho (CVM, 2014). Por sua vez, a diretoria deve controlar a ação de gestores, a fim de evitar más decisões de execução (FAMA; JENSEN 1983).

De fato, a qualidade da governança corporativa de uma empresa depende da gestão dos indivíduos que estão nos cargos de confiança. Além disso, também é importante a forma como esses líderes lidam com a administração na organização (CORREIA; AMARAL 2008). Essas ações devem buscar o aumento de riqueza dos acionistas. Segundo Hillman e Dalziel (2003), o conselho precisa de uma variedade de habilidades, informações e experiências para exercitar esse monitoramento de forma eficaz na empresa. Já Carter, Simkins e Simpson (2003) verificam que a diversidade de gênero aumenta a independência do conselho, o que melhora o monitoramento da organização. Tais fatos contribuem para o aumento de valor da empresa.

Por sua vez, a teoria do capital humano afirma que o investimento na formação pessoal melhora a produtividade dos trabalhadores. Gastos com formação, entre outros, podem ser considerados investimentos em capital. Referidos fatores produzem capital humano, uma vez que não é possível separar o indivíduo do seu conhecimento e habilidade. Assim, ao tomar decisões de investimento em capital humano, as empresas comparam a taxa de retorno desse investimento com os custos associados à sua contratação. (SCHULTZ, 1961; BECKER, 1964). 
Isidro e Sobral (2015) asseveram que a performance do conselho é afetada por sua diversidade, como um resultado da singularidade do capital humano que cada indivíduo tem. Segundo Andrade (2010), diferenças no estoque de capital humano são responsáveis por diferentes retornos na organização. Já para Peterson e Philpot (2017), homens e mulheres, em questão de educação e treinamento, são igualmente qualificados para as funções do conselho, todavia elas podem trazer um capital valioso para o conselho, afetando positivamente o valor da empresa. Segundo Metz (2015) e Lima et al. (2013), um dos traços da mulher é seu senso de preocupação com os outros. O fato de ela olhar com outros olhos para os que estão à sua volta faz com que seja ainda mais valorizada como capital humano. Esse recurso, quando bem investido, traz retornos positivos à empresa.

Por fim, a teoria da dependência de recursos sugere que os conselhos têm a importante função de facilitar o acesso a recursos vitais para o sucesso da organização (PFEFFER; SALANCIK, 1978). Essa teoria explica que a eficácia do conselho depende de seus recursos de capital humano, tais como gênero, educação, experiências gerenciais entre outras. Assim, empresas com mais diversidade de membros no conselho têm mais acesso a recursos que ajudam a reduzir a dependência externa, diminuir a incerteza e melhorar a reputação, o que leva ao aumento do valor da empresa.

Hillman e Dalziel (2003) declaram que a participação minoritária de mulheres no conselho de administração e diretoria estimula a divergência de pensamentos e motiva outros membros a considerar uma maior variedade de soluções potenciais. Além disso, Kim, Burns e Prescott (2009) sugerem que a diversidade do conselho aumenta a velocidade e capacidade dos gestores na tomada de decisões estratégicas. O conjunto desses fatos apontam para um melhor uso de seus recursos, agregando valor à empresa e criando mais riqueza aos seus proprietários.

As tabelas 1 e 2 apresentam uma síntese dos resultados dos estudos internacionais e nacionais, respectivamente, sobre o tema desta pesquisa. Em ambas as tabelas, a variável dependente é o valor da empresa, sendo representado pela proxy $Q$ de Tobin. Na tabela 1, verifica-se que a presença de mulheres na diretoria ou conselho de administração apresenta resultados inconclusivos sobre a criação de valor para a empresa. Há relações (não) significativas, sendo positivas e negativas. Com relação às variáveis de controle, tamanho apresenta uma relação significativa e negativa para a maioria dos estudos. Já para a alavancagem, verifica-se uma relação significativa e contrária ao esperado pela maioria dos estudos empíricos mencionados. Sobre a variável crescimento, os resultados não confirmam o esperado. Por fim, espera-se uma relação positiva ou negativa entre idade e criação de valor. A capacidade de a empresa adquirir experiências, habilidades e conhecimento no decorrer do tempo aponta para uma relação positiva. Entretanto, essa relação pode ser igualmente negativa, uma vez que a performance e o valor da empresa decrescem com o tempo.

Por sua vez, a tabela 2 indica que há menos estudos sobre o tema no Brasil que no exterior. À semelhança da tabela 1 , a presença de mulheres na diretoria ou conselho de administração também apresenta resultados inconclusivos sobre a criação de valor para a empresa, havendo resultados positivos e negativos (não) significativos. Sobre as variáveis de controle, destaca-se o sinal favorável aos demais estudos empíricos e significância estatística para a variável tamanho. Quanto à alavancagem e idade, os resultados não confirmam o esperado, enquanto a variável crescimento não é testada. Na revisão de referenciais empíricos, não são identificados estudos que analisem a relação entre presença das mulheres no conselho 
de administração/diretoria e o valor da empresa, sendo representado pelo índice market to book value. Ademais, em relação à hipótese referente à presença de mulheres no conselho de administração e diretoria e a melhora da performance financeira da empresa, foi encontrado apenas um único estudo que analisa a performance financeira como variável dependente. Tais fatos ratificam a relevância dessas análises nesta pesquisa.

Tabela 1 - Síntese de resultados dos estudos empíricos internacionais

\begin{tabular}{|c|c|c|c|c|c|c|c|c|c|c|c|c|c|}
\hline \multirow[t]{2}{*}{ Variáveis } & \multirow[t]{2}{*}{$\begin{array}{l}\text { Sinal } \\
\text { esp. }\end{array}$} & \multicolumn{2}{|c|}{$\begin{array}{c}\text { Nguyen e } \\
\text { Faff } \\
\text { (2007) }\end{array}$} & \multicolumn{2}{|c|}{$\begin{array}{c}\text { Adams e } \\
\text { Ferreira } \\
\text { (2009) }\end{array}$} & \multicolumn{2}{|c|}{$\begin{array}{c}\text { Loderer e } \\
\text { Waelchli } \\
\text { (2009) }\end{array}$} & \multicolumn{2}{|c|}{$\begin{array}{l}\text { Isidro e } \\
\text { Sobral } \\
\text { (2015) }\end{array}$} & \multicolumn{2}{|c|}{$\begin{array}{c}\text { Vintilã, } \\
\text { Onofrei e } \\
\text { Gherghina } \\
(2015)\end{array}$} & \multicolumn{2}{|c|}{$\begin{array}{c}\text { Toumi, } \\
\text { Benkraiem } \\
\text { e Hamrouni } \\
(2016)\end{array}$} \\
\hline & & Sinal & Sig & Sinal & Sig & Sinal & Sig & Sinal & Sig & Sinal & Sig & Sinal & Sig \\
\hline $\begin{array}{l}\text { Presença mulheres na } \\
\text { diretoria/conselho }\end{array}$ & + & + & $1 \%$ & - & $5 \%$ & $\mathrm{n} / \mathrm{d}$ & $\mathrm{n} / \mathrm{d}$ & - & $\mathrm{s} / \mathrm{s}$ & + & $10 \%$ & - & $1 \%$ \\
\hline Tamanho & - & - & $1 \%$ & $\mathrm{n} / \mathrm{d}$ & $n / d$ & - & $1 \%$ & - & $1 \%$ & + & $5 \%$ & - & $1 \%$ \\
\hline Alavancagem & - & $\mathrm{n} / \mathrm{d}$ & $\mathrm{n} / \mathrm{d}$ & $\mathrm{n} / \mathrm{d}$ & $n / d$ & $\mathrm{n} / \mathrm{d}$ & $\mathrm{n} / \mathrm{d}$ & - & $1 \%$ & + & $5 \%$ & + & $5 \%$ \\
\hline Crescimento & + & $\mathrm{n} / \mathrm{d}$ & $\mathrm{n} / \mathrm{d}$ & $\mathrm{n} / \mathrm{d}$ & $n / d$ & $\mathrm{n} / \mathrm{d}$ & $\mathrm{n} / \mathrm{d}$ & + & $\mathrm{s} / \mathrm{s}$ & - & $\mathrm{s} / \mathrm{s}$ & $\mathrm{n} / \mathrm{d}$ & $\mathrm{n} / \mathrm{d}$ \\
\hline Idade & + & $\mathrm{n} / \mathrm{d}$ & $\mathrm{n} / \mathrm{d}$ & $\mathrm{n} / \mathrm{d}$ & $n / d$ & - & $1 \%$ & - & $\mathrm{s} / \mathrm{s}$ & $\mathrm{n} / \mathrm{d}$ & $n / d$ & $\mathrm{n} / \mathrm{d}$ & $\mathrm{n} / \mathrm{d}$ \\
\hline Idade & - & $\mathrm{n} / \mathrm{d}$ & $\mathrm{n} / \mathrm{d}$ & $\mathrm{n} / \mathrm{d}$ & $n / d$ & - & $1 \%$ & - & $\mathrm{s} / \mathrm{s}$ & $\mathrm{n} / \mathrm{d}$ & $n / d$ & $\mathrm{n} / \mathrm{d}$ & $\mathrm{n} / \mathrm{d}$ \\
\hline
\end{tabular}

Notas: s/s: sem significância estatística e n/d: não disponível

Fonte: Elaborada pelos autores

Tabela 2 - Síntese de resultados dos estudos empíricos nacionais

\begin{tabular}{|c|c|c|c|c|c|c|c|c|c|}
\hline \multirow[t]{2}{*}{ Variáveis } & \multirow{2}{*}{$\begin{array}{c}\text { Sinal } \\
\text { esperado }\end{array}$} & \multicolumn{2}{|c|}{$\begin{array}{c}\text { Almeida, Klotzle e } \\
\text { Pinto (2013) }\end{array}$} & \multicolumn{2}{|c|}{ Oliveira (2013) } & \multicolumn{2}{|c|}{ Pereira (2013) } & \multicolumn{2}{|c|}{$\begin{array}{l}\text { Silva Junior e } \\
\text { Martins (2017) }\end{array}$} \\
\hline & & Sinal & Sig & Sinal & Sig & Sinal & Sig & Sinal & Sig \\
\hline $\begin{array}{l}\text { Presença mulheres na } \\
\text { diretoria/conselho }\end{array}$ & + & + & $\mathrm{s} / \mathrm{s}$ & - & $\mathrm{s} / \mathrm{s}$ & - & $1 \%$ & - & $\mathrm{s} / \mathrm{s}$ \\
\hline Tamanho & - & - & $1 \%$ & $\mathrm{n} / \mathrm{d}$ & $\mathrm{n} / \mathrm{d}$ & + & $5 \%$ & - & $1 \%$ \\
\hline Alavancagem & - & + & $\mathrm{s} / \mathrm{s}$ & $\mathrm{n} / \mathrm{d}$ & $\mathrm{n} / \mathrm{d}$ & + & $5 \%$ & - & $\mathrm{s} / \mathrm{s}$ \\
\hline Crescimento & + & $n / d$ & $\mathrm{n} / \mathrm{d}$ & $\mathrm{n} / \mathrm{d}$ & $\mathrm{n} / \mathrm{d}$ & $\mathrm{n} / \mathrm{d}$ & $\mathrm{n} / \mathrm{d}$ & $\mathrm{n} / \mathrm{d}$ & $n / d$ \\
\hline Idade & + & $\mathrm{n} / \mathrm{d}$ & $\mathrm{n} / \mathrm{d}$ & $\mathrm{n} / \mathrm{d}$ & $\mathrm{n} / \mathrm{d}$ & - & $\mathrm{s} / \mathrm{s}$ & $\mathrm{n} / \mathrm{d}$ & $\mathrm{n} / \mathrm{d}$ \\
\hline Idade & - & $\mathrm{n} / \mathrm{d}$ & $\mathrm{n} / \mathrm{d}$ & $\mathrm{n} / \mathrm{d}$ & $\mathrm{n} / \mathrm{d}$ & - & $\mathrm{s} / \mathrm{s}$ & $\mathrm{n} / \mathrm{d}$ & $\mathrm{n} / \mathrm{d}$ \\
\hline
\end{tabular}

Notas: s/s: sem significância estatística e n/d: não disponível

Fonte: Elaborada pelos autores

\section{METODOLOGIA}

A população de interesse consiste em todas as companhias não financeiras de capital aberto, listadas na B3 entre 2010 e 2017. A amostra final compreende 113 companhias, cuja evolução está apresentada na tabela 3. As bases de dados utilizadas são Capital IQ pertencente à Standard \& Poor's (S\&P), Economatica e Bloomberg. Os testes são realizados por meio do software econométrico Stata v.15.

\section{Tabela 3 - Evolução da amostra}

\begin{tabular}{lc}
\hline Descrição & Quantidade \\
\hline Amostra inicial & 267 \\
(-) Companhias sem valor de mercado de capital próprio ou negativo & 109 \\
(-) Companhias sem valor contábil de patrimônio líquido ou negativo & 29 \\
(-) Companhias sem dívida ou capital de terceiros de curto e longo prazos & 13 \\
(-) Intermediários financeiras (PagSeguro, Cielo, Card System) & 3 \\
(=) Amostra final & 113 \\
\hline
\end{tabular}

Fonte: Elaborada pelos autores 
A confirmação ou não das hipóteses ocorre via estatística descritiva, análise de correlação e regressão pooled cross section. As variáveis analisadas e sua descrição estão apresentadas no modelo de regressão da equação 1 e quadro 1, respectivamente. A princípio, apresenta-se a estatística descritiva dos dados que tem como objetivo a compreensão de populações e amostras. Entre as métricas da estatística descritiva há medidas de posição central e dispersão. Quanto às medidas de posição central, destacam-se a média aritmética, mediana e moda. Já entre as medidas de dispersão, ou de distanciamento em relação às de posição central, destacam-se amplitude, variância, desvio-padrão, mínimo e máximo (ANDERSON; SWEENEY; WILLIAMS, 2008; FÁVERO et al, 2009).

Na sequência, verifica-se o sinal, valor e significância estatística da correlação. A análise da correlação consiste no estudo entre duas variáveis e como elas se relacionam entre si, tendo como objetivo quantificar a força e direção da relação existente entre essas duas variáveis (ANDERSON, SWEENEY, WILLIAMS, 2008). Neste estudo, ela possui dois objetivos, a saber: verificar a existência de uma eventual correlação alta entre a variável dependente e independentes/controle e identificar uma eventual elevada multicolinearidade entre as variáveis independentes/controle. Caso ela exista, os testes do modelo de regressão devem confirmar esse fato.

Por fim, aplicam-se os modelos de regressão e testam-se seus pressupostos, sendo normalidade e homocedasticidade dos resíduos, linearidade dos parâmetros e ausência de alta multicolinearidade entre variáveis explicativas (GUJARATI, 2006; WOOLDRIDGE, 2007). De acordo com Gujarati (2006), existem três categorias de dados que podem ser utilizados para análises empíricas: dados cross section, dados pooled cross section e dados em painel. Este estudo utiliza o modelo de regressão com dados pooled cross section, modelo no qual se pressupõe que o coeficiente angular $\beta$ da variável explicativa $X$ é idêntico para todas as observações ao longo do tempo (FÁVERO et al, 2009).

As equações 1, 2 e 3 apresentam os modelos de regressão com dados em pooled cross section desta pesquisa, cujas variáveis estão descritas no quadro 1 e são obtidas a partir dos estudos empíricos apresentados nas tabelas 1 e 2 . As equações 1 e 2 têm como referência proxies de criação de valor na empresa, enquanto a equação 3 possui como variável dependente a performance financeira da companhia.

$$
\begin{aligned}
& \text { VEQT it }=\beta_{0}+\beta_{1} P M U_{i t}+\beta_{2} T A M_{i t}+\beta_{3} A L A_{i t}+\beta_{4} C R E_{i t}+\beta_{5} I D A_{i t}+\varepsilon_{i t} \\
& V E M B_{i t}=\beta_{0}+\beta_{1} P_{M U} U_{i t}+\beta_{2} T A M_{i t}+\beta_{3} A_{L} A_{i t}+\beta_{4} C R E_{i t}+\beta_{5} I D A_{i t}+\varepsilon_{i t} \\
& P F_{i t}=\beta_{0}+\beta_{1} P M U_{i t}+\beta_{2} T A M_{i t}+\beta_{3} A L A_{i t}+\beta_{4} C R E_{i t}+\beta_{5} I D A_{i t}+\varepsilon_{i t}
\end{aligned}
$$

Em que: $i=$ empresa, $t=$ tempo; $\beta_{0}=$ coeficiente angular, $\beta_{1}$ a $_{5}=$ coeficientes lineares e $\varepsilon=$ termo de erro da equação 


\section{Quadro 1 - Descrição das variáveis}

\begin{tabular}{|c|c|c|c|c|c|c|c|}
\hline Sigla & Descrição & Tipo & Sinal & Fórmula & Componente & Referências & Fontes \\
\hline VEQT & $\begin{array}{l}\text { Valor da } \\
\text { empresa } \\
\text { com Q de } \\
\text { Tobin }\end{array}$ & $\mathrm{D}$ & $\mathrm{n} / \mathrm{a}$ & $\begin{array}{l}\text { VEQT = } \\
\text { QTobin = } \\
(\text { VMPL + } \\
\text { VCDT }) / A T\end{array}$ & $\begin{array}{l}\text { VMPL = valor de mercado } \\
\text { do patrimônio líquido = } \\
\text { (número de ações em } \\
\text { circulação x preço das } \\
\text { ações) } \\
\text { VCDT = valor contábil das } \\
\text { dívidas de curto e longo } \\
\text { prazos } \\
\text { AT = ativo total }\end{array}$ & $\begin{array}{l}\text { Nguyen e Faff } \\
\text { (2007); García- } \\
\text { Meca, Martínez- } \\
\text { Ferrero e } \\
\text { García-Sánchez } \\
\text { (2014); Isidro e } \\
\text { Sobral (2015) }\end{array}$ & $\mathrm{CIQ}$ \\
\hline VEMB & $\begin{array}{l}\text { Valor da } \\
\text { empresa } \\
\text { com } \\
\text { market to } \\
\text { book value }\end{array}$ & $\mathrm{D}$ & $\mathrm{n} / \mathrm{a}$ & $\begin{array}{l}\text { VEMB = VMPL } \\
/ V C P L\end{array}$ & $\begin{array}{l}\text { VMPL = valor de mercado } \\
\text { do patrimônio líquido = } \\
\text { (número de ações em } \\
\text { circulação x preço das } \\
\text { ações) } \\
\text { VCPL = Valor contábil do } \\
\text { patrimônio líquido }\end{array}$ & $\begin{array}{l}\text { Greene e Jame } \\
\text { (2013); Bastos e } \\
\text { Nakamura } \\
\text { (2009); Bastos, } \\
\text { Basso e } \\
\text { Nakamura } \\
(2009)\end{array}$ & $\mathrm{CIQ}$ \\
\hline PF & $\begin{array}{l}\text { Perfor- } \\
\text { mance } \\
\text { financeira }\end{array}$ & $\mathrm{D}$ & $\mathrm{n} / \mathrm{a}$ & $\begin{array}{l}P F=R O A= \\
E B I T / A T\end{array}$ & $\begin{array}{l}\text { ROA = Retorno sobre o ativo } \\
\text { EBIT = Lucro antes dos juros } \\
\text { e impostos } \\
\text { AT = ativo total }\end{array}$ & $\begin{array}{l}\text { Bathula (2008); } \\
\text { Khudzari e Sori } \\
(2012)\end{array}$ & $\mathrm{CIQ}$ \\
\hline PMU & $\begin{array}{l}\text { Presença } \\
\text { de } \\
\text { mulheres } \\
\text { na diretoria } \\
\text { e conselho }\end{array}$ & I & + & $\begin{array}{l}P M U=N M D C \\
/ T M D C\end{array}$ & $\begin{array}{l}\text { NMDC = número de } \\
\text { mulheres na diretoria e } \\
\text { conselho de administ. } \\
\text { TMDC = total de membros } \\
\text { da diretoria e conselho }\end{array}$ & $\begin{array}{l}\text { Pucheta- } \\
\text { Martínez e Bel- } \\
\text { Oms (2015); } \\
\text { Sila, González e } \\
\text { Hagendorff } \\
\text { (2016) }\end{array}$ & $\mathrm{B}$ \\
\hline TAM & Tamanho & $\mathrm{C}$ & - & $T A M=L n(A T)$ & $\begin{array}{l}\mathrm{Ln}=\text { logaritmo neperiano } \\
\mathrm{AT}=\text { ativo total }\end{array}$ & $\begin{array}{l}\text { Silva Junior e } \\
\text { Martins (2017); } \\
\text { Pucheta- } \\
\text { Martínez e Bel- } \\
\text { Oms (2015) }\end{array}$ & $\mathrm{CIQ}$ \\
\hline ALA & $\begin{array}{l}\text { Alavan- } \\
\text { cagem }\end{array}$ & $\mathrm{C}$ & - & $\begin{array}{l}A L A=\mathrm{VCDT} / \\
A T\end{array}$ & $\begin{array}{l}\text { VCDT = valor contábil das } \\
\text { dívidas de curto e longo } \\
\text { prazos } \\
\text { AT = ativo total }\end{array}$ & $\begin{array}{l}\text { Dezsö e Ross } \\
(2012) ; \text { Toumi, } \\
\text { Benkraiem e } \\
\text { Hamrouni } \\
\text { (2016); Silva } \\
\text { Junior e Martins } \\
\text { (2017) }\end{array}$ & $\mathrm{CIQ}$ \\
\hline CRE & $\begin{array}{l}\text { Cresci- } \\
\text { mento }\end{array}$ & $\mathrm{C}$ & + & $\begin{array}{l}C R E=\Delta \% R L V \\
/ 3\end{array}$ & $\begin{array}{l}\Delta \% R L V=\text { variação \% ou } \\
\text { média do crescimento } \\
\text { percentual da RLV dos } 3 \\
\text { últimos anos } \\
\text { RLV = receita líquida de } \\
\text { vendas }\end{array}$ & $\begin{array}{l}\text { Greene e Jame } \\
\text { (2013); Isidro e } \\
\text { Sobral (2015) }\end{array}$ & $\begin{array}{l}\mathrm{ClQ} \text { e } \\
\mathrm{E}\end{array}$ \\
\hline IDA & Idade & $\mathrm{C}$ & $+/-$ & $I D A=\operatorname{Ln}(N A)$ & $\begin{array}{l}\text { Ln = logaritmo neperiano } \\
\mathrm{NA}=\text { número de anos de } \\
\text { existência da empresa }\end{array}$ & $\begin{array}{l}\text { Isidro e Sobral } \\
(2015)\end{array}$ & $\mathrm{CIQ}$ \\
\hline
\end{tabular}

Em que: $D$ = variável dependente, $I$ = variável independente, $C=$ variável de controle das equações 1,2 e 3 , $\mathrm{B}=$ Bloomberg, $\mathrm{CIQ}=$ Capital IQ (S\&P) e E = Economatica 


\section{ANÁLISE DOS RESULTADOS}

A estatística descritiva apresentada na tabela 4 indica que as variáveis apresentam dados para todos os anos da amostra. O valor da empresa, calculado via $Q$ de Tobin (VEQT), aponta que a estrutura de capital da companhia - a valor de mercado - em média, se aproxima do valor contábil de seus ativos. Já o valor da empresa - obtido via comparação entre o valor de mercado e contábil do capital próprio da empresa (VEMB) - apresenta uma média duas vezes maior que VEQT. Isso sinaliza uma expectativa de criação de valor no futuro. Com relação à performance financeira $(\mathrm{PF})$ ou retorno sobre o ativo da companhia, sua média é $7 \%$, havendo variações em torno de resultados negativos de $45 \%$ e positivos de $47 \%$. Quanto ao tamanho (TAM), verifica-se que em média as empresas são de grande porte, possuindo $\mathrm{R} \$ 14,6$ bilhões de total de ativo. Cabe observar que o valor da tabela está apresentado em logaritmo neperiano. Quanto à alavancagem (ALA), em média, as companhias têm $32 \%$ da sua estrutura de capital sendo financiada por terceiros. Já a média de crescimento das receitas líquidas (CRE) a cada triênio é de $28 \%$. Por fim, em média, as empresas possuem 53,5 anos de existência. Na tabela, esse valor está igualmente apresentado em logaritmo neperiano.

Tabela 4 - Estatística descritiva

\begin{tabular}{cccccc}
\hline Variáveis & Observações & Média & Desvio padrão & Mínimo & Máximo \\
\hline VEQT & 904 & 1,105653 & 0,750515 & 0,162383 & 6,591313 \\
VEMB & 904 & 2,026193 & 2,396005 & 0,022138 & 23,24403 \\
PF & 904 & 0,071073 & 0,068038 & $-0,456450$ & 0,472921 \\
TAM & 904 & 8,564601 & 1,380292 & 5,028475 & 12,75288 \\
ALA & 904 & 0,329647 & 0,159203 & 0,000653 & 0,767496 \\
CRE & 904 & 0,289875 & 1,369470 & $-0,440059$ & 23 \\
IDA & 904 & 3,733850 & 0,751737 & 1,098612 & 4,976734 \\
\hline
\end{tabular}

Fonte: Elaborada pelos autores

Quanto à presença de mulheres na diretoria e conselho de administração das companhias (PMU), a tabela 5 apresenta sua evolução ao longo dos anos da amostra. Essa variável possui uma quantidade de observações inferior às demais apresentadas na tabela 3 , sendo de 760 no total ou de 95 companhias para cada ano da amostra. Logo, há 18 companhias sem dados em um total de 113. Em média, as mulheres possuem participação de apenas $4,1 \%$ nos conselhos de administração e diretoria, ainda que haja uma evolução oscilante ao longo dos anos. Entretanto, destaca-se que uma alta quantidade de companhias não possui representantes do gênero feminino nessas entidades de governança - média de 70 companhias ou $73,4 \%$ do total de 113 . Quanto ao máximo de $100 \%$, isso ocorre em uma única companhia no ano de 2016 , reforçando o caráter de excepcionalidade da participação de mulheres na diretoria e conselho de administração das companhias 
Anie Levinbuk Schmiliver, Marcella Scaraficci Teixeira, Mariana Domingues Brandão, Vitória Dantas Andrade, Michele Nascimento Jucá

Tabela 5 - Evolução da presença de mulheres nas companhias

\begin{tabular}{lccccccccc}
\hline Descrição & $\mathbf{2 0 1 0}$ & $\mathbf{2 0 1 1}$ & $\mathbf{2 0 1 2}$ & $\mathbf{2 0 1 3}$ & $\mathbf{2 0 1 4}$ & $\mathbf{2 0 1 5}$ & $\mathbf{2 0 1 6}$ & $\mathbf{2 0 1 7}$ & Total \\
\hline Quantidade de observações & 95 & 95 & 95 & 95 & 95 & 95 & 95 & 95 & 760 \\
Média de participação \% & 2,7 & 3,6 & 3,8 & 4,8 & 4,3 & 3,8 & 5,7 & 4,3 & 4,1 \\
Quant. com 0\% de particip. & 76 & 73 & 70 & 67 & 68 & 67 & 67 & 70 & n/a \\
Quant. com 100\% de partic. & 0 & 0 & 0 & 0 & 0 & 0 & 1 & 0 & n/a \\
Quant. com dados difer. 0 e 1 & 19 & 22 & 25 & 28 & 27 & 28 & 27 & 25 & n/a \\
\hline
\end{tabular}

Nota: $n / a=$ não aplicável

Fonte: Elaborada pelos autores

Por sua vez, a tabela 6 apresenta a análise de correlação entre as variáveis dos modelos das equações 1, 2 e 3 . As correlações entre VEQT e VEMB com PMU são positivas e significativas, o que confirma previamente a $\mathrm{H}_{1}$ : A presença de mulheres no conselho de administração e diretoria aumenta o valor da empresa. O mesmo resultado é obtido para a correlação entre PF e PMU, ratificando a $\mathrm{H}_{2}$ : A presença de mulheres no conselho de administração e diretoria melhora a performance financeira da empresa. Tais resultados estão alinhados com a teoria institucional.

Quanto às variáveis de controle, verifica-se uma correlação negativa entre TAM e ALA com VEQT, conforme esperado pela teoria de dependência de recursos e de agência, respectivamente. Essa relação é a mesma obtida entre ALA e VEQT. Já a correlação positiva e significativa entre IDA e VEQT/VEMB confirma a teoria de dependência de recursos. Crescimento não apresenta significância estatística nas correlações. Além disso, destacam-se as correlações significativas negativa e positiva entre ALA e IDA com PF, respectivamente, estando em linha com os resultados das variáveis dependentes de criação de valor (VEQT e VEMB). Por fim, as correlações positivas e significativas entre as variáveis explicativas não são altas, indicando uma possível baixa multicolinearidade entre elas.

Tabela 6 - Análise de correlação

\begin{tabular}{|c|c|c|c|c|c|c|c|c|}
\hline Variáveis & VEQT & VEMB & PF & PMU & TAM & ALA & CRE & IDA \\
\hline VEQT & 1,0000 & & & & & & & \\
\hline VEMB & $\begin{array}{c}0,7401 \\
{[\mathbf{0}, \mathbf{0 0 0 0}]}\end{array}$ & 1,0000 & & & & & & \\
\hline PF & $\begin{array}{c}0,6225 \\
{[\mathbf{0}, \mathbf{0 0 0 0}]}\end{array}$ & $\begin{array}{c}0,4812 \\
{[\mathbf{0}, \mathbf{0 0 0 0}]}\end{array}$ & 1,0000 & & & & & \\
\hline PMU & $\begin{array}{c}0,1926 \\
{[0.0000]}\end{array}$ & $\begin{array}{c}0,1426 \\
{[0,00011}\end{array}$ & $\begin{array}{c}0,0887 \\
{[0,0145]}\end{array}$ & 1,0000 & & & & \\
\hline TAM & $\begin{array}{c}-0.1372 \\
{[\mathbf{0}, \mathbf{0 0 0 0 ]}}\end{array}$ & $\begin{array}{l}-0,0579 \\
{[0,0821]}\end{array}$ & $\begin{array}{c}0,0419 \\
{[0,2082]}\end{array}$ & $\begin{array}{c}0,2224 \\
{[0,0000]}\end{array}$ & 1,0000 & & & \\
\hline ALA & $\begin{array}{c}-0,0666 \\
{[\mathbf{0}, \mathbf{0 4 5 3}]}\end{array}$ & $\begin{array}{c}0,1351 \\
{[\mathbf{0}, \mathbf{0 0 0 0}]}\end{array}$ & $\begin{array}{c}-0,0865 \\
{[\mathbf{0 , 0 0 9 2}]}\end{array}$ & $\begin{array}{c}0,0856 \\
{[\mathbf{0 , 0 1 8 2}]}\end{array}$ & $\begin{array}{c}0,2159 \\
{[\mathbf{0}, \mathbf{0 0 0 0}]}\end{array}$ & 1,0000 & & \\
\hline CRE & $\begin{array}{c}-0,0046 \\
{[0,8892]}\end{array}$ & $\begin{array}{l}-0,0035 \\
{[0,9173]}\end{array}$ & $\begin{array}{l}-0,0450 \\
{[0,1768]}\end{array}$ & $\begin{array}{c}-0,0014 \\
{[0,9690]}\end{array}$ & $\begin{array}{c}-0,0651 \\
{[0,0505]}\end{array}$ & $\begin{array}{c}-0,0005 \\
{[0,9871]}\end{array}$ & 1,0000 & \\
\hline IDA & $\begin{array}{c}0,0856 \\
{[\mathbf{0}, \mathbf{0 1 0 0}]}\end{array}$ & $\begin{array}{c}0,0805 \\
{[0,0115]}\end{array}$ & $\begin{array}{c}0,0882 \\
{[\mathbf{0}, 0080]}\end{array}$ & $\begin{array}{c}0,0114 \\
{[0,7530]}\end{array}$ & $\begin{array}{c}0,0444 \\
{[0,1819]}\end{array}$ & $\begin{array}{c}-0,1343 \\
{[\mathbf{0}, \mathbf{0 0 0 1}]}\end{array}$ & $\begin{array}{c}-0,2203 \\
{[\mathbf{0}, \mathbf{0 0 0 0}]}\end{array}$ & 1,0000 \\
\hline
\end{tabular}

Notas: Os valores de cima referem-se ao coeficiente de Pearson, enquanto os de baixo, ou seja, os que estão entre colchetes apresentam sua significância estatística. Os valores em negrito são os que possuem significância estatística no valor da correlação aos níveis de $1 \%$ ou $5 \%$.

Fonte: Elaborada pelos autores 
A tabela 7 apresenta os resultados dos testes de regressão cross section pooled para os modelos 1 , 2 e 3 , que correspondem às equações 1,2 e 3 . Os testes dos pressupostos de todos eles identificam que não há normalidade nos resíduos. Entretanto, o teorema de Gauss-Markov mostra que, mesmo sem a normalidade dos resíduos, os estimadores dos mínimos quadrados ordinários (MQO) ainda são os melhores estimadores lineares não viesados (MELNVs) (ANDERSON et al, 2008). Além disso, o valor do fator de inflação de variância (VIF) é inferior a 2. Tal fato indica a inexistência de alta multicolinearidade entre as variáveis explicativas. Já o teste de Breusch Pagan indica a presença de heterocedasticidade nos resíduos, sendo corrigida por meio de erros-padrão robustos. Quanto à linearidade dos coeficientes, ela é verificada pela significância do modelo econométrico como um todo, com o teste $\mathrm{F}$ tendo um $\mathrm{p}$-valor de $0 \%$.

Tabela 7 - Regressão com pooled cross section

\begin{tabular}{|c|c|c|c|c|c|c|}
\hline \multirow{2}{*}{ Variáveis } & \multicolumn{2}{|c|}{ Modelo 1 (VEQT | H } & \multicolumn{2}{|c|}{ Modelo 2 (VEMB | H } & \multicolumn{2}{|c|}{ Modelo 3 (PF | H } \\
\hline & Coef. & p-valor & Coef. & p-valor & Coef. & p-valor \\
\hline PMU & 2,216531 & $0,000 * * *$ & 4,816503 & 0,001 *** & 0,072669 & $0,017^{* *}$ \\
\hline TAM & $-0,098149$ & $0,000 * * *$ & $-0,250802$ & $0,000 * * *$ & 0,002234 & 0,233 \\
\hline ALA & $-0,240334$ & 0,161 & 2,775292 & $0,000 * * *$ & $-0,049035$ & $0,002 * * *$ \\
\hline CRE & 0,002066 & 0,847 & 0,016094 & 0,789 & $-0,002994$ & 0,117 \\
\hline IDA & 0,080835 & 0,025 ** & 0,260469 & 0,012 ** & 0,003377 & 0,343 \\
\hline Cons. & 1,652493 & $0,000 * * *$ & 2,212185 & $0,002 * * *$ & 0,056119 & $0,009 * * *$ \\
\hline F p-valor & \multicolumn{2}{|c|}{$0,0000 * * *$} & \multicolumn{2}{|c|}{$0,0000^{* * *}$} & \multicolumn{2}{|c|}{$0,0009 * * *$} \\
\hline $\mathrm{R}^{2}$ ajustado & \multicolumn{2}{|c|}{0,0736} & \multicolumn{2}{|c|}{0,0541} & \multicolumn{2}{|c|}{0,0206} \\
\hline $\begin{array}{l}\text { No. de } \\
\text { observações }\end{array}$ & \multicolumn{2}{|c|}{760} & \multicolumn{2}{|c|}{760} & \multicolumn{2}{|c|}{760} \\
\hline
\end{tabular}

Os modelos 1 e 2 confirmam a $\mathrm{H}_{1}$ : A presença de mulheres no conselho de administração e diretoria aumenta o valor da empresa, assim como o modelo 3 confirma a $\mathrm{H}_{2}$ : A presença de mulheres no conselho de administração e diretoria melhora a performance financeira da empresa. Dessa forma, a comprovação de $\mathrm{H}_{1}$ ratifica as teorias institucional e de dependência de recursos, enquanto a constatação de $\mathrm{H}_{2}$ corrobora as teorias de agência e do capital humano.

A teoria institucional discute a forma como as ações de uma organização podem ser influenciadas por fatores externos e sociais. Já a teoria de dependência de recursos sugere que a diversidade de recursos de capital humano explica a eficácia do conselho. No caso da $\mathrm{H}_{1}$, os resultados obtidos estão alinhados com os estudos de Nguyen e Faff (2007) e de Vintilã, Onofrei e Gherghina (2015) - vide tabela 1. De fato, a cada 1\% de aumento da presença de mulheres na diretoria e conselho de administração, há um aumento de 2,21\% no valor da empresa, se calculado via $Q$ de Tobin (VEQT), e de 4,81\%, se calculado via relação entre valor de mercado e contábil (VEMB) do capital próprio da empresa.

Quanto à performance financeira (PF), a teoria de agência sugere que a presença de mecanismos de governança corporativa mitiga os conflitos entre principais e agentes, aumentando o resultado financeiro da organização. Já a teoria do capital humano afirma que o investimento na formação pessoal melhora a produtividade e desempenho dos seus colaboradores. Essa performance é afetada pela diversidade dos 
gestores em cargos executivos, sobretudo. Uma das principais características das mulheres é seu senso de preocupação com os demais. O valor do coeficiente da variável PMU, na equação 3 . indica que a cada $1 \%$ de aumento na presença de mulheres na diretoria e conselho de administração, há um aumento no retorno sobre o ativo da empresa (ROA) de 0,07\%.

O coeficiente linear ou constante é significante em todos os modelos. Já tamanho (TAM) apresenta uma relação negativa e significativa com ambas as variáveis de criação de valor (VEQT e VEMB), ratificando a teoria de dependência de recursos. Quanto à alavancagem (ALA), verifica-se uma relação negativa e significativa com performance financeira. Assim, a cada $1 \%$ de aumento no seu endividamento, a empresa reduz seu ROA em $0,04 \%$. Por fim, verifica-se uma relação positiva e significativa entre idade (IDA) e VEQT/VEMB.

\section{CONCLUSÃO}

O conselho de administração e diretoria das companhias constituem importante mecanismo de governança corporativa, cuja eficácia depende de seus recursos de capital humano, incluindo gênero, conforme explicitado pela teoria da dependência de recursos. Assim sendo, este estudo busca compreender se a presença de mulheres nesses instrumentos de governança aumenta o valor e performance financeira das companhias. Os testes aplicados são os de estatística descritiva, análise de correlação e regressão cross section com dados empilhados ou pooled. A amostra final é composta por 113 companhias listadas na B3, cujos dados são obtidos a partir das bases da Bloomberg, Capital IQ e Economatica, entre os anos de 2010 e 2017.

Como resultado, tem-se a confirmação das hipóteses 1 e 2. No caso de $\mathrm{H}_{1}$ - A presença de mulheres no conselho de administração e diretoria aumenta o valor da empresa, há uma ratificação das teorias institucional e de dependência de recursos. Por sua vez, a verificação de $\mathrm{H}_{2}$ - A presença de mulheres no conselho de administração e diretoria melhora a performance financeira da empresa - corrobora as teorias de agência e do capital humano. Quanto às variáveis de controle, tamanho, alavancagem e idade mostram-se igualmente significantes e de acordo com os sinais esperados.

Entre as limitações apresentadas neste estudo, destaca-se a baixa quantidade de informações relacionadas à presença de mulheres no conselho de administração e diretoria. Para fins de sua evolução, sugere-se, além da ampliação da amostra, a inclusão de outras variáveis relacionadas às teorias mencionadas, tais como a relação entre política de compensação e gênero na gestão das organizações, bem como a identificação dos segmentos de listagem especiais de governança corporativa na B3 - novo mercado, nível 1 e 2. 


\section{REFERÊNCIAS}

ADAMS, B.; FERREIRA, D. Women in the boardroom and their impact on governance and performance. Journal of Financial Economics, v.94, n.2, p. 291-309, 2009

ALMEIDA, R. S.; KLOTZLE, M. C.; PINTO, A. C. F. Composição do conselho de administração no setor de energia elétrica do Brasil. Revista de Administração da UNIMEP, v. 11, n. 1, p. 156-180, 2013.

ANDERSON, D R; SWEENEY D J; WILLIAMS T A. Estatística aplicada à administração e economia. São Paulo: Cengage Learning, 2008

ANDRADE, R. A teoria do capital humano e a qualidade da educação nos estados brasileiros. Rio Grande do Sul: UFRGS, 2010. Monografia (Faculdade de Ciências Econômicas) - Universidade Federal do Rio Grande do Sul, Porto Alegre, Rio Grande do Sul, 2010. Disponível em <http://www.lume.ufrgs.br/handle/10183/25425>. Acesso em: 01 abril 2018.

BASTOS D. D.; BASSO L. F. C.; NAKAMURA W. T. Determinantes da estrutura de capital das companhias abertas na América Latina: um estudo empírico considerando fatores macroeconômicos e institucionais. Revista de Administração Mackenzie, v.10, n.6, p. 47-77, 2009.

BASTOS D. D.; NAKAMURA W. T. Determinantes da estrutura de capital das companhias abertas no Brasil, México e Chile no período 2001-2006. Revista Contabilidade \& Finanças, USP, v.20, n.50, p. 75-94, 2009.

BATHULA, H. Board characteristics and firm performance: Evidence from New Zealand. Auckland: Auckland University of Technology, 2008. Tese de Doutorado (PhD em Filosofia) - Auckland University of Technology, Nova Zelândia, 2008. Disponível em <http://aut.researchgateway.ac.nz/bitstream/handle/10292/376/BathulaH. pdf?sequence=4\&isAllowed=y> . Acesso em: 22 junho 2018

BEAUVOIR, S. O segundo sexo: fatos e mitos. Rio de Janeiro: Ed. Nova Fronteira, 1980

BECKER, G. Human capital: a theoretical and empirical analysis, with special reference to education. Chicago: University of Chicago Press, 1964.

BILIMORIA, D. Building the business case for women corporate directors. Journal of Business Ethics, v.14, n.1, p. 25-40, 2000.

BLUM, T. C.; FIELDS, D. L. Organization-level determinants of women in management. Academy of Management Journal, v.37, n.2, p. $241-269,1994$

BRASIL. Decreto lei $\mathrm{n}^{\circ}$ 6.404, de 15 de dezembro de 1976. Diário Oficial da União. Disponível em <http://www.planalto.gov.br/ccivil_03/leis/L6404consol.htm>. Acesso em: 26 de março de 2018.

BURKE, R. J. Women on corporate boards of directors: views of Canadian chief executive officers. Women in Management Review, v.9, n.5, p. 3-10, 1994

BOSCIA, V.; MARCHETTI, P.; STEFANELLI, V. Do the women matter in co-operative banks' boards? Journal of Governance and Regulation, v.4, n.3, p. 57-66, 2015

BYRON, K.; POST, C. Women on boards and firm financial performance: a meta-analysis Corporate Governance: An International Review. v.24, n.4, p. 428-442, 2016

CAMPBELL, K.; MINGUEZ-VERA, A. Gender diversity in the boardroom and firm financial performance. Journal of Business Ethics, v.83, n.3, p. 435-451, 2008

CARDOSO, A; HANASHIRO, D.M.M. Percepção masculina sobre as barreiras das mulheres executivas. Pretexto, v. 19, n.1, p.73-89, 2018

CARTER, D.; SIMKINS, B.; SIMPSON, W. Corporate governance, board diversity, and firm value. Financial Review, v.38, n.1, p. 33-53, 2003

COMISSÃO DE VALORES MOBILIÁRIOS (CVM). O mercado de valores mobiliários brasileiro. Rio de Janeiro: Comissão de Valores Mobiliários, 2014.

COOK, A.; GLASS C. Women on corporate boards: do they advance corporate social responsibility? 2017. Disponível em: <https://doi.org/10.1177/0018726717729207>. Acesso em: 01 abril 2018.

CORREIA, L. F; AMARAL, H. F. Arcabouço teórico para os estudos de governança corporativa: os pressupostos subjacentes à teoria da agência. Revista de Gestão, v.15, n.3, p. 1-10, 2008.

DAILY, C. M.; DALTON, D. R. Women in the boardroom: a business imperative. The Journal of Business Strategy, v.24, n.5, p.8-9, 2003

DEPARTAMENTO INTERSINDICAL DE ESTATÍSTICA E ESTUDOS SOCIOECONÔMICOS (DIEESE). Estudos e Pesquisa. Mulheres 2013. A inserção das mulheres nos mercados de trabalho metropolitanos e a desigualdade nos rendimentos. Disponível em: <https://www.dieese.org.br/analiseped/2013/2013pedmulhermet.pdf>. Acesso em: 18 abril 2018 
DEZSO, C; ROSS, D G. Does female representation in top management improve firm performance? A panel data investigation. 2012. Disponível em: <http://ssrn.com/abstract=1088182>. Acesso em: 01 abril 2018.

DIMAGGIO, P. J.; POWELL, W. W. The iron cage revisited: institutional isomorphism and collective rationality in an organizational field. American Sociological Review, v.48, n.2, p. 147-160, 1983

FAMA, F.; JENSEN, M. Agency problems and residual claims. Journal of Law and Economics, v.26, n.2, p.288-307, 1983.

FÁVERO, L. P; BELFLORE, P. P; SILVA F. L; CHAN B. L. Análise de dados: modelagem multivariada para a tomada de decisões. Rio de Janeiro: Elsevier, 2009.

FERREIRA, D. Board diversity. In: BAKER, H. K.; ANDERSON, R. Corporate governance: a synthesis of theory, research, and practice, USA: John Willey, 2010, chapter 12.

GARCIA-MECA, E; GARCIA SANCHEZ, I M; MARTINEZ-FERRERO, J. Board diversity and its effects on bank performance: An international analysis. Journal of Banking \& Finance, Elsevier, v.53, n.C, p. 202-214, 2015

GREENE, T., JAME, R. Company name fluency, investor recognition, and firm value. Journal of Financial Economics, v.109, n.3, p. 813-834, 2011.

GUJARATI, D; PORTER D C. Econometria básica. São Paulo: AMGH, 5. ed., 2012.

HILLMAN, A.; DALZIEL, T. Boards of directors and firm performance: integrating agency and resource dependence perspectives. Academy of Management Review, v.28, n.3, p.383-396, 2003.

INSTITUTO BRASILEIRO DE GOVERNANÇA CORPORATIVA (IBGC). Código das boas práticas para reuniões do conselho de administração. São Paulo, 2010. Disponível em <http://www.ibgc.org.br/userfiles/9.pdf>. Acesso em: 26 março 2018.

INSTITUTO BRASILEIRO DE GOVERNANÇA CORPORATIVA (IBGC). Código das Melhores Práticas de Governança Corporativa. v.4, São Paulo, 2009. Disponível em <http://www.ibgc.org.br/userfiles/Codigo_julho_2010_a4.pdf>. Acesso em: 04 abril 2018.

INSTITUTO BRASILEIRO DE GOVERNANÇA CORPORATIVA (IBGC). Guia de orientação para o gerenciamento de riscos corporativos. Série Cadernos de Governança Corporativa, v.3, São Paulo, 2007. Disponível em <http://www.ibgc.org.br/userfiles/3.pdf>. Acesso em: 25 março 2018.

INSTITUTO BRASILEIRO DE GOVERNANÇA CORPORATIVA (IBGC). IBGC incentiva a presença de mulheres na governança corporativa. 2015. Disponível em <http://www.ibgc.org.br/index.php/19412/IBGC-incentiva-a-presenca-demulheres-na-Governanca-Corporativa>. Acesso em: 30 março 2018.

ISIDRO, H.; SOBRAL, M. The effects of women on corporate boards on firm value, financial performance, and ethical and social compliance. Journal of Business Ethics, v.132, n.1, p. 1-19, 2015.

JENSEN, M. C.; MECKLING, W. H. Theory of the firm: managerial behavior, agency costs, and ownership structure. Journal of Financial Economics, v. 3, n. 4, p. 78-79, 1976.

KHUDZARI, J.; 'SORI Z.M. Gender diversity in the boardroom and firm performance of Malaysian public listed companies. Procedia - Social and Behavioral Sciences, v. 65, n.1, p. 1077-1085, 2012

KIM, B.; BURNS, M.; PRESCOTT, J. The strategic role of the board: The impact of board structure on top management team strategic action capability. Corporate Governance: An International Review, v.17, n.6, p.728-743, 2009.

KIM, D.; STARKS, L. T. Gender diversity on corporate boards: do women contribute unique skills? American Economic Review, v.106, n.5, p. 267-71, 2016.

KLÜCK, S. J. Diversity in the board of directors - the effect of woman on firm performance and the gender quota law: a twofold empirical research. Faculty of Economics and Business, University of Amsterdam, 2012. Disponível em: $<$ http://www.scriptiesonline.

uba.uva.nl/cgi/b/bib/bibidx?c=uvascript;type=simple;view=reslist;fmt=long;rgn1=id;q1=oai\%3aarno\%3a443247;sid=b0f9d166a650fc548bafdecac 041c4c7; sort=publicationyear;cc=uvascript;page=reslist;start=1;size=10;lang=en>. Acesso em: 04 abril 2018.

LIMA, G.S.; CARVALHO NETO, A; LIMA, M.S; TANURE, B.; VERSIANI, F. O teto de vidro das executivas brasileiras. Pretexto, v. 14, n. 4, p. 65-80, 2013.

LODERER, C.; WAELCHLI, U. Firm age and performance. Discussion paper series in economics and management. Disponível em: <http://www.geaba.de/wp-content/uploads/ 2017/07/DP-09-18.pdf>. Acesso em: 20 abril 2018.

LUCENA, C. A. A teoria do capital humano: história, trabalho e capacitação dos trabalhadores. In: CONGRESSO DE PESQUISA E ENSINO EM EDUCAÇÃO, v.2, 2003. Uberlândia. Anais, Uberlândia, 2003.

LOW, D.C.M.; ROBERTS, H.; WHITING, R.H. Board gender diversity and firm performance: Empirical evidence from Hong Kong, South Korea, Malaysia and Singapore. Pacific-Basin Finance Journal, v.35-A, p.381-401, 2015. Disponível em: <https://www.sciencedirect.com/science/article/ pii/S0927538X15000311>. Acesso em: 26 junho 2018. 
MADALOZZO, R. CEOs e composição do conselho de administração: a falta de identificação pode ser motivo para existência de teto de vidro para mulheres no Brasil? Revista de Administração Contemporânea, v.15, n.1, p. 126-137, 2011.

METZ, E.S. Gestão Feminina: A presença das mulheres na liderança de empresas. Ágora - Revista de Divulgação Científica, v.19 n.2, p.169-178, 2014

MEYER J. M; ROWAN, B. Institutionalized organizations: formal structure as myth and ceremony. American Journal of Sociology, v.83, n.2, p. 340-363, 1977.

NGUYEN, H.; FAFF, R. Impact of board size and diversity on firm value: Australian evidence. Corporate Ownership \& Control, v.4, n.2, p. 24-31, 2007.

NIELSEN, S.; HUSE, M. The contribution of women on boards of directors: going beyond the surface. Corporate Governance: An International Review. v.18, n.2, p. 136-148, 2010.

OLIVEIRA, C de. A participação feminina nos conselhos de administração e o desempenho corporativo: um estudo sobre as empresas listadas na BM\&FBOVESPA de 2002 a 2011. São Leopoldo: Universidade do Vale do Rio dos Sinos. Dissertação de mestrado (Faculdade de Ciências Contábeis) - Universidade do Vale do Rio dos Sinos, São Leopoldo, 2013

<http://www.repositorio.jesuita.org.br/bitstream/handle/UNISINOS/3182/Carine\%20de\%20Oliveira.pdf?sequence=1\&isAll owed=y>. Acesso em: 01 Maio 2018.

PEREIRA, J. Mulheres nos conselhos de administração medida social ou fonte de valor? Porto: Universidade do Porto, 2013. Dissertação de mestrado (Faculdade de Ciências Econômicas) - Universidade do Porto, Porto, Portugal, 2013. Disponível em: $<$ https://www.google.com/url? sa $=$ \&\&ct=j\&q=\&esrc=s\&source=web\&cd=1\&ved=0ahUKEwjjs4Tf2aPbAhUGhpAKHd62DrsQFggoMAA\&url=https \%3A\%2 F\%2Fsigarra.up.pt\%2Ffep\%2Fpt\%2Fpub_geral.show_file\%3Fpi_gdoc_id\%3D142488\&usg=AOvVaw2Fn6tOMEv0HkIJH f9C1A-r>. Acesso em 19 abril 2018.

PETERSON, C.; PHILPOT, J. Women's roles on U.S. Fortune 500 boards: director expertise and committee memberships. Journal of Business Ethics, v.72. n.2, p. 177-196, 2017.

PFEFFER, J.; SALANCIK, G. R. The external control of organizations: a resource dependence perspective. New York: Stanford University Press, 1978.

PUCHETA-MARTINEZ, M C; BEL-OMS, I. The board of directors and dividend policy: the effect of gender diversity. Industrial and Corporate Change, v.25, n.3, p. 523-547, 2016

SANTOS, R. V.; RIBEIRO, E. P. Diferenciais de rendimentos entre homens e mulheres no Brasil revisitado: explorando o "teto de vidro". 2009. Disponível em: <http://www.ie.ufrj.br/eventos/ seminarios/pesquisa/texto06_05_02.pdf>. Acesso em: 01 abril 2018.

SCHULTZ, T. W. Investment in human capital. The American Economic Review, v. 51, n. 1, p. 1-17, 1961.

SILA, V; GONZALEZ, A. HAGENDORFF, J. Women on board: Does boardroom gender diversity affect firm risk? Journal of Corporate Finance, v.36, n.3, p.26-53, 2016

SILVA JÚNIOR, C. P; MARTINS, O. S. Mulheres no conselho afetam o desempenho financeiro? Uma análise da representação feminina nas empresas listadas na BM\&FBovespa. Sociedade, Contabilidade e Gestão, v. 12, n. 1, p. 6276, 2017.

SUDECK, K.; IATRIDIS, G. Female board appointments and stock market reactions: evidence from the German stock market. Investment Management and Financial Innovation, v.11, n.3, p.73-80, 2014.

TOUMI, N. BENKRAIEM, R; HAMROUNI, A. Board director disciplinary and cognitive influence on corporate value creation. Corporate Governance, v.16, n.3, p. 564-578, 2016

VINTILÃ, G; ONOFREI, M; GHERGHINA, C. The effects of corporate board and CEO characteristics on firm value: empirical evidence from listed companies on the Bucharest stock exchange. Taylor \& Francis Journals, v.51, n.6, p. 12441260, 2015

WOOLDRIDGE, J. M. Introdução à econometria: uma abordagem moderna. São Paulo: Thomson, 2007. 